\title{
Quantum Monte Carlo Study of a Positron in an Electron Gas
}

\author{
N. D. Drummond \\ Department of Physics, Lancaster University, Lancaster LA1 4YB, United Kingdom and TCM Group, Cavendish Laboratory, \\ University of Cambridge, J. J. Thomson Avenue, Cambridge CB3 OHE, United Kingdom \\ P. López Ríos and R. J. Needs \\ TCM Group, Cavendish Laboratory, University of Cambridge, J. J. Thomson Avenue, Cambridge CB3 OHE, United Kingdom \\ C. J. Pickard \\ Department of Physics, University College London, Gower Street, London WC1E 6BT, United Kingdom
} (Received 28 April 2011; published 8 November 2011)

\begin{abstract}
Quantum Monte Carlo calculations of the relaxation energy, pair-correlation function, and annihilatingpair momentum density are presented for a positron immersed in a homogeneous electron gas. We find smaller relaxation energies and contact pair-correlation functions in the important low-density regime than predicted by earlier studies. Our annihilating-pair momentum densities have almost zero weight above the Fermi momentum due to the cancellation of electron-electron and electron-positron correlation effects.
\end{abstract}

DOI: 10.1103/PhysRevLett.107.207402

PACS numbers: 78.70.Bj, 02.70.Ss, 71.10.Ca, 71.60.+Z

Electron-positron annihilation underlies both medical imaging with positron emission tomography (PET) and studies of materials using positron annihilation spectroscopy (PAS) [1]. Positrons entering a material rapidly thermalize and the majority annihilate with opposite-spin electrons to yield pairs of photons at energies close to $0.511 \mathrm{MeV}$. In a PET scan, positrons are emitted by radionuclides in biologically active tracer molecules and the resulting annihilation radiation is measured to image the tracer concentration. The interaction of low-energy positrons with molecules is therefore of substantial experimental and theoretical interest [2]. PAS is used to investigate microstructures in metals, alloys, semiconductors, insulators [1], polymers [3], and nanoporous materials [4]. Positrons are repelled by the positively charged nuclei and tend to become trapped in voids within the material. The positron lifetime is measured as the interval between the detection of a photon emitted in the $\beta^{+}$radioactive decay that produces the positron and the detection of the annihilation radiation [1]. The lifetime is characteristic of the region in which the positron settles, and PAS is a sensitive, nondestructive technique for characterizing the size, location, and concentration of voids in materials. Measuring the Doppler broadening of the annihilation radiation or the angular correlation between the two $0.511 \mathrm{MeV}$ photons yields information about the momentum density (MD) of the electrons in the presence of the positron. These techniques may be used to investigate the Fermi surfaces of metals [5].

The aim of PAS experiments is to investigate a host material without the changes induced by the positron. The positron is, however, an invasive probe which polarizes the electronic states of the material. Disentangling the properties of the host from the changes induced by the positron is a major theoretical challenge. Positrons in condensed matter may be modeled with two-component density functional theory (DFT) [6], in which the correlations are described by a functional of the electron and positron density components. Within the local density approximation (LDA), this functional is obtained from the difference $\Delta \Omega$ between the energy of a homogeneous electron gas (HEG) with and without an immersed positron. $\Delta \Omega$ is known as the relaxation energy, and is equal to the electron-positron correlation energy.

Two-component DFT gives reasonable electron and positron densities, but the DFT orbitals do not describe electron-positron correlation properly [6,7]. The electronpositron pair-correlation function (PCF) $g(r)$ and the annihilating-pair momentum density (APMD) $\rho(\bar{p})$ constructed from the DFT orbitals are therefore poor. The contact PCF $g(0)$ is particularly important because it determines the annihilation rate $\lambda=3 g(0) /\left(4 c^{3} r_{s}^{3}\right)$ [1] for a positron immersed in a paramagnetic HEG, where $r_{s}$ is the electron density parameter and $c$ is the speed of light in vacuo [8]. If the electron and positron motions were uncorrelated $g(0)$ would be unity, but the strong correlation leads to much larger values, particularly at low densities, where an electron-positron bound state (positronium or Ps) or even an electron-electron-positron bound state $\left(\mathrm{Ps}^{-}\right)$ may be formed.

We have used the variational and diffusion quantum Monte Carlo (VMC and DMC) methods $[9,10]$ as implemented in the CASINO code [11] to study a single positron in a HEG. Fermionic antisymmetry is imposed via the fixed-node approximation, in which the nodal surface is constrained to equal that of a trial wave function. We used 
Slater-Jastrow (SJ) and Slater-Jastrow-backflow (SJB) trial wave functions $[12,13]$. The latter go beyond the singleparticle SJ nodal surface by replacing the particle coordinates in the Slater determinants by "quasiparticle coordinates." SJB wave functions give the highest accuracy obtained to date for the HEG $[12,13]$. We also tested two types of orbitals: (i) plane-wave orbitals for each particle and (ii) orbitals which describe the pairing between the electrons and positron. The pairing orbitals were obtained from mean-field calculations performed in the reference frame of the positron, so the orbitals are functions of the separation of an electron and the positron [14]. Within this impurity-frame DFT (IF-DFT) method, the pairing orbitals describe the electron-positron correlation quite well on their own [14] and give a different nodal surface from the plane-wave orbitals. (NB, our quantum Monte Carlo calculations were performed in the laboratory frame.) The four wave-function forms used are

$$
\begin{aligned}
& \Psi_{\mathrm{PW}}^{\mathrm{SJ}}=e^{J(\mathbf{R})}\left[e^{i \mathbf{k}_{i} \cdot \mathbf{r}_{\uparrow}}\right]\left[e^{i \mathbf{k}_{j} \cdot \mathbf{r}_{\downarrow}}\right], \\
& \Psi_{\mathrm{PW}}^{\mathrm{SJB}}=e^{J(\mathbf{R})}\left[e^{i \mathbf{k}_{i} \cdot\left(\mathbf{r}_{\uparrow}+\xi(\mathbf{R})\right)}\right]\left[e^{i \mathbf{k}_{j} \cdot\left(\mathbf{r}_{\downarrow}+\xi(\mathbf{R})\right)}\right], \\
& \Psi_{\text {pair }}^{\mathrm{SJ}}=e^{J(\mathbf{R})}\left[\phi_{i}\left(\mathbf{r}_{\uparrow}-\mathbf{r}_{p}\right)\right]\left[\phi_{j}\left(\mathbf{r}_{\downarrow}-\mathbf{r}_{p}\right)\right], \\
& \Psi_{\text {pair }}^{\mathrm{SJB}}=e^{J(\mathbf{R})}\left[\phi_{i}\left(\mathbf{r}_{\uparrow}-\mathbf{r}_{p}+\boldsymbol{\xi}(\mathbf{R})\right)\right]\left[\phi_{j}\left(\mathbf{r}_{\downarrow}-\mathbf{r}_{p}+\boldsymbol{\xi}(\mathbf{R})\right)\right],
\end{aligned}
$$

where $\mathbf{R}$ denotes the positions of all the particles, $\mathbf{r}_{\uparrow}$ and $\mathbf{r}_{\downarrow}$ denote the positions of up- and down-spin electrons, respectively, $\mathbf{r}_{p}$ is the positron position, and $[\cdots]$ denotes a Slater determinant. The Jastrow exponent $J(\mathbf{R})$ [15] and the backflow displacement $\boldsymbol{\xi}(\mathbf{R})$ [13] contain parameters that were optimized separately for each wave function and system. The Jastrow exponents were first optimized using the efficient VMC variance-minimization scheme of Ref. [16], and then all the parameters (including the backflow parameters) were optimized together using the VMC energy-minimization scheme of Ref. [17]. The pairing orbitals $\left\{\phi_{i}\right\}$ were represented using $B$-spline functions on a real-space grid [18]. The electron-positron cusp condition was enforced on the pairing orbitals for wave function $\Psi_{\text {pair }}^{\mathrm{SJ}}[19,20]$; for the other three wave functions, the cusp conditions were imposed via the Jastrow factor. In all our calculations the simulation-cell Bloch vector [21] was chosen to be $\mathbf{k}_{s}=\mathbf{0}$.

Tests at high $\left(r_{s}=1\right)$ and low $\left(r_{s}=8\right)$ electron densities show that the qualitative features of the variations in $\Delta \Omega, g(r)$, and $\rho(\bar{p})$ with $r_{s}$ are the same for each of the wave functions of Eq. (1). However, as shown in the Supplemental Material [22], we obtained lower VMC and DMC energies with the SJB wave functions $\left(\Psi_{\mathrm{PW}}^{\mathrm{SJB}}\right.$ and $\left.\Psi_{\text {pair }}^{\mathrm{SJB}}\right)$ than the SJ ones $\left(\Psi_{\mathrm{PW}}^{\mathrm{SJ}}\right.$ and $\left.\Psi_{\text {pair }}^{\mathrm{SJ}}\right)$, and therefore we used SJB wave functions to obtain all our main results. The pairing orbitals give lower SJB-VMC energies than the plane-wave orbitals, but the SJB-DMC energies with the plane-wave and pairing orbitals are almost identical [the same to within error bars at $r_{s}=8$, and differing by only $0.0013(5)$ a.u. at $r_{s}=1$ with 54 electrons]. The lack of sensitivity to the orbitals used, and hence the nodal surface, suggests that the DMC energies are highly accurate. The energies reported in this Letter are from DMC calculations using wave function $\Psi_{\mathrm{PW}}^{\mathrm{SJB}}$. Such calculations are considerably less expensive than calculations using $\Psi_{\text {pair }}^{\text {SJB }}$ due to (i) the lower energy variance achieved with $\Psi_{\mathrm{PW}}^{\mathrm{SJB}}[22]$ and (ii) the fact that plane-wave orbitals are cheaper to evaluate. The DMC energies were extrapolated to zero time step. Our production DMC calculations were performed in cells containing $N=54$ electrons. Tests of convergence with respect to system size up to $N=114$ electrons are described in the Supplemental Material [22]. The cell volume was chosen to be $(N-1)(4 / 3) \pi r_{s}^{3}$, so that the electron density far from the positron was correct. IFDFT calculations [14] suggest that finite-size effects due to the interaction of images of the positron are negligible for $N \geq 54$ electrons. The uncertainty in the DMC relaxation energy due to finite-size errors ranges from about \pm 0.01 a.u. at $r_{s}=1$ to \pm 0.003 a.u. at $r_{s}=8$. At low density the estimated finite-size errors in our data are an order of magnitude smaller than the difference of about 0.04 a.u. between our SJB-DMC data and the BorońskiNieminen result [6].

Our DMC relaxation energies are plotted in Fig. 1 and are well fitted by the form

$$
\Delta \Omega\left(r_{s}\right)=\frac{A_{-1} r_{s}^{-1}+A_{0}+A_{1} r_{s}-0.262005 B_{2} r_{s}^{2}}{1+B_{1} r_{s}+B_{2} r_{s}^{2}},
$$

where $A_{-1}=-0.260361, \quad A_{0}=-0.261762, \quad A_{1}=$ $0.00375534, \quad B_{1}=0.113718$, and $B_{2}=0.0270912$. Equation (2) tends to the correct low-density limit of the energy of the $\mathrm{Ps}^{-}$ion [23]. Equation (2) does not yield the exact high-density behavior of the random phase approximation (RPA), although this is only relevant for $r_{s}<0.1$ [24]. VMC energies for a positron in a HEG have been reported previously [25], but we have used superior trial

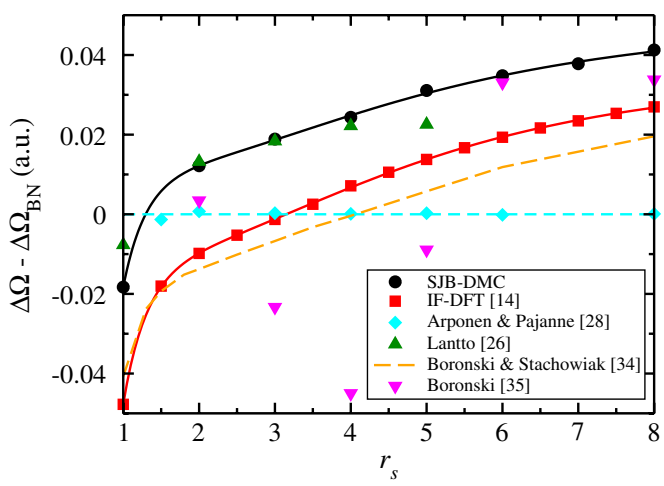

FIG. 1 (color online). Relaxation energy against density parameter from our SJB-DMC calculations and other studies $[14,26,28,34,35]$, relative to the Boronski-Nieminen expression, $\Delta \Omega_{\mathrm{BN}}[6]$ (horizontal dashed line). 
wave functions and have obtained very different results. At high densities our relaxation energies are similar to those of Lantto [26], but at lower densities we obtain smaller values. The SJB-DMC and IF-DFT results [14] and the data of Ref. [27] show similar behavior with $r_{s}$, while the Boronski-Nieminen fit [6] to the data of Ref. [28] is markedly different. The Boroński-Nieminen [6] expression for $\Delta \Omega\left(r_{s}\right)$ is widely used in two-component DFT calculations, but our study suggests it is not very accurate and should be replaced by Eq. (2).

We calculated the APMD using the VMC method with an optimized SJB trial wave function with pairing orbitals $\left(\Psi_{\text {pair }}^{\text {SJB }}\right.$, because these give lower VMC energies than plane-wave orbitals $\left(\Psi_{\mathrm{PW}}^{\mathrm{SJB}}\right)$. These calculations were performed by constraining an electron and the positron to lie on top of one another throughout the simulation [22]. APMDs at different densities are plotted in Fig. 2, with the normalization chosen such that $\int_{0}^{\infty} 4 \pi \bar{p}^{2} \rho(\bar{p}) d \bar{p}=$ $(4 / 3) \pi k_{F}^{3}$. Our results clearly show the enhancement of the APMD below the Fermi momentum predicted by Kahana [29], but our data differ quantitatively from previous results $[14,27,29]$. Our VMC data have almost no
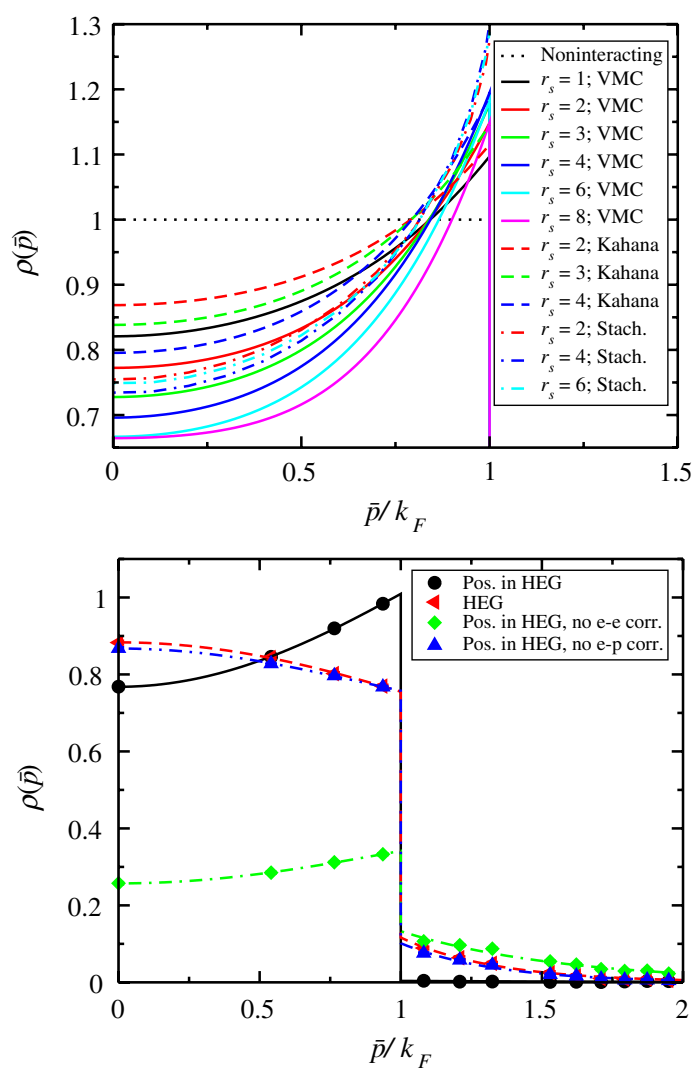

FIG. 2 (color online). Top: APMDs $[\rho(\bar{p})]$ for different densities. The solid lines show our VMC data obtained with wave function $\Psi_{\text {pair }}^{\mathrm{SJB}}$ and $N=114$ electrons, while the dashed and dash-dotted lines show the data of Kahana [29] and Stachowiak [27], respectively. Bottom: APMDs for the positron in HEG and the HEG at $r_{s}=8$ and $N=54$ electrons, calculated using $\Psi_{\mathrm{PW}}^{\mathrm{SJB}}$. weight above the Fermi momentum over the entire density range studied, even though the weight in the MD above $k_{F}$ in the HEG is substantial at low densities. For example, we find that the APMD immediately above $k_{F}$ is roughly $10 \%$ of the value for the HEG at $r_{s}=1$ and $3 \%$ at $r_{s}=8$.

Suppression of the weight in the APMD above $k_{F}$ was demonstrated theoretically by Carbotte and Kahana [30], but our study gives a more detailed and accurate picture. We investigated the weight above $k_{F}$ using VMC calculations with the wave function $\Psi_{\mathrm{PW}}^{\mathrm{SJB}}$ by selectively eliminating interparticle correlations. Neglecting electron-electron and electron-positron correlations gives the familiar "top hat" MD of the noninteracting system. Calculations with the electron-positron terms removed give an APMD indistinguishable from the MD of the HEG, with a tail above $k_{F}$. Calculations including electron-positron correlation but neglecting electron-electron correlation show Kahana enhancement below $k_{F}$ and a tail above $k_{F}$. When, however, both electron-electron and electron-positron correlations are included, the tail above $k_{F}$ is largely suppressed, as shown in the lower panel of Fig. 2.

The suppression of the tail in the APMD can be explained by examining the behavior of the two-body terms in the Jastrow exponent. (For simplicity, we consider the $\Psi_{\mathrm{PW}}^{\mathrm{SJ}}$ wave function in the following discussion.) The Jastrow exponent $J(\mathbf{R})$ is the sum of electron-electron $\left[u_{\uparrow \uparrow}(r)\right.$ and $u_{\uparrow \downarrow}(r)$, where the arrows indicate spins] and electron-positron $\left[u_{\mathrm{ep}}(r)\right]$ terms. If one assumes that

$$
u_{\uparrow \downarrow}(r)=u_{\Uparrow}(r)=-u_{\mathrm{ep}}(r)
$$

then the APMD has exactly zero weight above $k_{F}$, as shown in the Supplemental Material [22]. The RPA (linear response theory) shows that Eq. (3) holds at large $r$ and the Kato cusp conditions force the gradients of $u_{\uparrow \downarrow}(r)$ and $u_{\mathrm{ep}}(r)$ to satisfy Eq. (3) at $r=0$. The cusp conditions for parallel and antiparallel spin electrons are different and therefore $u_{\uparrow \downarrow}(r)$ and $u_{\uparrow}(r)$ must differ at small $r$, but antisymmetry ensures that the probability of parallel-spin electrons being closer than $r_{s}$ is small. As shown in the Supplemental Material [22], plots of the terms in the Jastrow exponent demonstrate the approximate validity of Eq. (3).

We calculated the PCFs within VMC and DMC using $\Psi_{\mathrm{PW}}^{\mathrm{SJB}}$ wave functions, because these give almost identical results to pairing orbitals, but the calculations are much cheaper [22]. The final results were evaluated by extrapolated estimation (twice the DMC PCF minus the VMC PCF) [31], in order to eliminate the leading-order errors. In Fig. 3, the electron-positron contact PCF $g(0)$ is plotted relative to the Boronski-Nieminen form [6]. Our contact $\mathrm{PCF}$ data are well represented by

$$
\begin{aligned}
g(0)= & 1+1.23 r_{s}+a_{3 / 2} r_{s}^{3 / 2}+a_{2} r_{s}^{2}+a_{7 / 3} r_{s}^{7 / 3} \\
& +a_{8 / 3} r_{s}^{8 / 3}+0.173694 r_{s}^{3},
\end{aligned}
$$




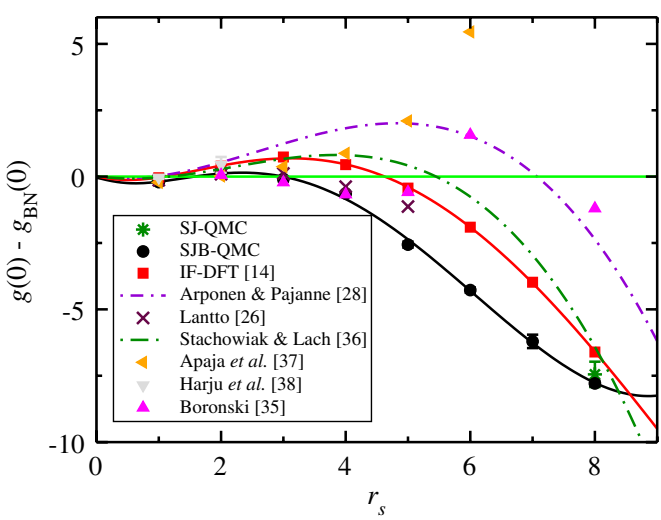

FIG. 3 (color online). Deviation of the contact PCF $g(0)$ from the form $g_{\mathrm{BN}}(0)$ of Boroński and Nieminen [6] together with other results in the literature [14,26,28,35-38].

where $a_{3 / 2}=-3.38208, a_{2}=8.6957, a_{7 / 3}=-7.37037$, and $a_{8 / 3}=1.75648$. Equation (4) satisfies the highdensity (RPA) [24] and low-density ( $\left.\mathrm{Ps}^{-}\right)$limiting behaviors [23]. The $\mathrm{Ps}^{-}$limit appears to be approached slowly. Our full data for $g(r)$ are given in the Supplemental Material [22]. The IF-DFT data follow the extrapolated SJB data quite well, while the other many-body calculations give somewhat larger values of $g(r)$ at low densities. In the density range $r_{s}=5-8$ a.u., our values of $g(0)$ are approximately $9 \%$ smaller than those given by the Boroński-Nieminen expression [6]. The local increase of the electron density around the positron caused by their mutual attraction is modeled in two-component DFT using an "enhancement factor" based on data for $g(0)$. Using our smaller values of $g(0)$ would reduce the enhancement factor and hence the overestimation of annihilation rates obtained with the positronic LDA [32].

In conclusion, our results are the most accurate obtained so far for a positron in a HEG. Our data for $\Delta \Omega$ are sufficient to define the energy functional for a twocomponent positronic DFT within the LDA. They would also be useful in developing semilocal [33] or other functionals. Our PCF data give a smaller enhancement factor than the standard Boroński-Nieminen expression [6]. Twocomponent DFT, using our electron-positron correlation functional and enhancement factor, could be used to predict the annihilation rate of low densities of positrons at defects in real materials, to aid the interpretation of PAS experiments, although such calculations may be complicated by the need to describe orthopositronium formation. Our APMDs have very little weight above $k_{F}$ because of the cancellation of electron-electron and electron-positron correlation effects. We have derived an exact result relating Eq. (3) to the complete absence of weight in the APMD $\rho(\bar{p})$ for $\bar{p}>k_{F}$.

We acknowledge financial support from the U.K. Engineering and Physical Sciences Research Council (EPSRC). Computer resources were provided by the
Cambridge High-Performance Computing Facility and the Lancaster High-End Computing cluster.

[1] R. Krause-Rehberg and H.S. Leipner, Positron Annihilation in Semiconductors (Springer-Verlag, Berlin, 1999).

[2] G. F. Gribakin, J. A. Young, and C. M. Surko, Rev. Mod. Phys. 82, 2557 (2010).

[3] R. A. Pethrick, Prog. Polym. Sci. 22, 1 (1997).

[4] D. W. Gidley, H.-G. Peng, and R. S. Vallery, Annu. Rev. Mater. Res. 36, 49 (2006).

[5] Zs. Major et al., Phys. Rev. Lett. 92, 107003 (2004).

[6] E. Boroński and R. M. Nieminen, Phys. Rev. B 34, 3820 (1986).

[7] M. J. Puska and R. M. Nieminen, Rev. Mod. Phys. 66, 841 (1994).

[8] We use Hartree atomic units $\left(\hbar=|e|=m_{e}=4 \pi \epsilon_{0}=1\right)$ throughout.

[9] D. M. Ceperley and B. J. Alder, Phys. Rev. Lett. 45, 566 (1980).

[10] W. M. C. Foulkes et al., Rev. Mod. Phys. 73, 33 (2001).

[11] R. J. Needs et al., J. Phys. Condens. Matter 22, 023201 (2010).

[12] Y. Kwon, D. M. Ceperley, and R. M. Martin, Phys. Rev. B 58, 6800 (1998).

[13] P. López Ríos et al., Phys. Rev. E 74, 066701 (2006).

[14] N.D. Drummond et al., Phys. Rev. B 82, 035107 (2010).

[15] N. D. Drummond, M. D. Towler, and R. J. Needs, Phys. Rev. B 70, 235119 (2004).

[16] N. D. Drummond and R. J. Needs, Phys. Rev. B 72, 085124 (2005).

[17] C. J. Umrigar et al., Phys. Rev. Lett. 98, 110201 (2007).

[18] D. Alfè and M. J. Gillan, Phys. Rev. B 70, 161101 (2004).

[19] A. Ma et al., J. Chem. Phys. 122, 224322 (2005).

[20] S. J. Binnie et al., Phys. Rev. B 82, 165431 (2010).

[21] G. Rajagopal et al., Phys. Rev. B 51, 10591 (1995).

[22] See Supplemental Material at http://link.aps.org/ supplemental/10.1103/PhysRevLett.107.207402 for plots of the relaxation energy and PCF, a demonstration that Eq. (3) in the main text leads to the cancellation of the postedge tail in the APMD, and some additional technical details about our quantum Monte Carlo calculations.

[23] A. M. Frolov, Phys. Lett. A 342, 430 (2005).

[24] J. Arponen, J. Phys. C 11, L739 (1978).

[25] G. Ortiz, Ph.D. thesis, Swiss Federal Institute of Technology, Lausanne, 1992. The relevant data from this thesis are also reported in Refs. [34,37].

[26] L. J. Lantto, Phys. Rev. B 36, 5160 (1987).

[27] H. Stachowiak, Phys. Rev. B 41, 12522 (1990).

[28] J. Arponen and E. Pajanne, Ann. Phys. (N.Y.) 121, 343 (1979).

[29] S. Kahana, Phys. Rev. 129, 1622 (1963).

[30] J.P. Carbotte and S. Kahana, Phys. Rev. 139, A213 (1965).

[31] D. M. Ceperley and M. H. Kalos, in Monte Carlo Methods in Statistical Physics, edited by K. Binder (SpringerVerlag, Heidelberg, 1979), 2nd ed., p. 145. 
[32] J. Mitroy and B. Barbiellini, Phys. Rev. B 65, 235103 (2002).

[33] B. Barbiellini et al., Phys. Rev. B 53, 16201 (1996).

[34] E. Boroński and H. Stachowiak, Phys. Rev. B 57, 6215 (1998).

[35] E. Boroński, Europhys. Lett. 75, 475 (2006).
[36] H. Stachowiak and J. Lach, Phys. Rev. B 48, 9828 (1993).

[37] V. Apaja, S. Denk, and E. Krotscheck, Phys. Rev. B 68, 195118 (2003).

[38] A. Harju et al., J. Radiol. Nucl. Chem. 211, 193 (1996). 